\title{
NUMERICAL RADIUS INEQUALITIES FOR SEVERAL OPERATORS
}

\author{
OMAR HIRZALLAH and FUAD KITTANEH
}

\section{Abstract}

Let $A, B, X$, and $A_{1}, \ldots, A_{2 n}$ be bounded linear operators on a complex Hilbert space. It is shown that

$$
w\left(\sum_{k=1}^{2 n-1} A_{k+1}^{*} X A_{k}+A_{1}^{*} X A_{2 n}\right) \leq 2\left(\sum_{k=1}^{n}\left\|A_{2 k-1}\right\|^{2}\right)^{1 / 2}\left(\sum_{k=1}^{n}\left\|A_{2 k}\right\|^{2}\right)^{1 / 2} w(X)
$$

and

$$
w(A B \pm B A) \leq 2 \sqrt{2}\|B\| \sqrt{w^{2}(A)-\frac{\left|\|\operatorname{Re} A\|^{2}-\|\operatorname{Im} A\|^{2}\right|}{2}},
$$

where $w(\cdot)$ and $\|\cdot\|$ are the numerical radius and the usual operator norm, respectively. These inequalities generalize and refine some earlier results of Fong and Holbrook. Some applications of our results are given.

\section{Introduction}

Let $\mathscr{H}$ be a complex Hilbert space with inner product $\langle\cdot, \cdot\rangle$, and let $\mathfrak{B}(\mathscr{H})$ be the space of all bounded linear operators on $\mathscr{H}$. The numerical radius of an operator $X \in \mathfrak{B}(\mathscr{H})$, denoted by $w(X)$, is defined by

$$
w(X)=\sup _{\|x\|=1}|\langle X x, x\rangle| .
$$

It is well-known that $w(\cdot)$ defines a norm on $\mathscr{B}(\mathscr{H})$, which is equivalent to the usual operator norm $\|\cdot\|$. Namely, for $X \in \mathfrak{B}(\mathscr{H})$, we have

$$
\frac{1}{2}\|X\| \leq w(X) \leq\|X\| .
$$

There are some important properties of the numerical radius (see, e.g., [3]) such as its weak unitary invariance

$$
w\left(U^{*} X U\right)=w(X)
$$

Received 23 October 2011. 
for every unitary $U \in \mathfrak{B}(\mathscr{H})$, and the fact that it satisfies the power inequality

$$
w\left(X^{n}\right) \leq(w(X))^{n}
$$

for $n=1,2, \ldots$

In [6] Fong and Holbrook have established two remarkable numerical radius inequalities for operators. These inequalities say that if $A, B, X \in \mathfrak{B}(\mathscr{H})$, then

$$
w\left(A^{*} X+X A\right) \leq 2\|A\| w(X)
$$

and

$$
w(A B+B A) \leq 2 \sqrt{2}\|B\| w(A) .
$$

Recent generalizations of the inequality (1.1) have been given in [4] and [5].

In this paper, we are interested in further analysis of these numerical radius inequalities. In Section 2, we establish a generalization of the inequality (1.1) to several operators. In Section 3, we present a general numerical radius inequality form which a refinement of the inequality (1.2) follows as a special case.

\section{A generalization of the inequality (1.1) to several operators}

In this section, we present a generalization of the Fong-Holbrook inequality (1.1) to several operators. In order to achieve our goal, we need the following lemma [6].

Lemma 2.1. Let $X \in \mathfrak{B}(\mathscr{H})$, and let $x_{1}, \ldots, x_{n} \in \mathscr{H}$. Then

$$
\left|\sum_{k=1}^{n-1}\left\langle X x_{k}, x_{k+1}\right\rangle\right| \leq\left(\sum_{k=1}^{n}\left\|x_{k}\right\|^{2}\right) w(X) .
$$

Based on Lemma 2.1, we have the following result. This result will play a central role in our generalization of the inequality (1.1).

Lemma 2.2. Let $X \in \mathfrak{B}(\mathscr{H})$, and let $x_{1}, \ldots, x_{n} \in \mathscr{H}$. Then

$$
\left|\sum_{k=1}^{n-1}\left\langle X x_{k}, x_{k+1}\right\rangle+\left\langle X x_{n}, x_{1}\right\rangle\right| \leq\left(\sum_{k=1}^{n}\left\|x_{k}\right\|^{2}\right) w(X) .
$$

Proof. Let $m$ be a natural number, and define a sequence of vectors $\left(y_{k}\right)_{k=1}^{m n+1}$ in $\mathscr{H}$ by

$$
y_{i n+j}= \begin{cases}x_{j}, & i=0, \ldots, m-1, j=1, \ldots, n \\ x_{1}, & i=m, j=1 .\end{cases}
$$


Then

$$
\sum_{k=1}^{m n+1}\left\|y_{k}\right\|^{2}=m \sum_{k=1}^{n}\left\|x_{k}\right\|^{2}+\left\|x_{1}\right\|^{2}
$$

and

$$
\sum_{k=1}^{m n}\left\langle X y_{k}, y_{k+1}\right\rangle=m\left(\sum_{k=1}^{n-1}\left\langle X x_{k}, x_{k+1}\right\rangle+\left\langle X x_{n}, x_{1}\right\rangle\right) .
$$

Applying the inequality (2.1) to the sequence $\left(y_{k}\right)_{k=1}^{m n+1}$, we have

$$
\left|\sum_{k=1}^{m n}\left\langle X y_{k}, y_{k+1}\right\rangle\right| \leq\left(\sum_{k=1}^{m n+1}\left\|y_{k}\right\|^{2}\right) w(X) .
$$

It follows from the identities (2.3), (2.4) and the inequality (2.5) that

$$
\left|\sum_{k=1}^{n-1}\left\langle X x_{k}, x_{k+1}\right\rangle+\left\langle X x_{n}, x_{1}\right\rangle\right| \leq \frac{m \sum_{k=1}^{n}\left\|x_{k}\right\|^{2}+\left\|x_{1}\right\|^{2}}{m} w(X) .
$$

Now, the desired inequality follows from the inequality (2.6) by letting $m \rightarrow$ $\infty$.

An application of Lemma 2.2 can be seen in the following result.

Proposition 2.3. Let $X \in \mathfrak{B}(\mathscr{H})$, and let $\tilde{X}$ be the $n \times n$ operator matrix in $\mathfrak{B}\left(\oplus_{k=1}^{n} \mathscr{H}\right)$ that has the operator $X$ in the subdiagonal and in the top righthand corner in the position $(1, n)$. Then

$$
w(\tilde{X})=w(X) .
$$

In particular, for $n=2,3$, and 4 , we have

$$
w\left(\left[\begin{array}{cc}
0 & X \\
X & 0
\end{array}\right]\right)=w\left(\left[\begin{array}{ccc}
0 & 0 & X \\
X & 0 & 0 \\
0 & X & 0
\end{array}\right]\right)=w\left(\left[\begin{array}{cccc}
0 & 0 & 0 & X \\
X & 0 & 0 & 0 \\
0 & X & 0 & 0 \\
0 & 0 & X & 0
\end{array}\right]\right)=w(X) .
$$

Proof. Let $y=\left[y_{1}, \ldots, y_{n}\right]^{T}$ be a unit vector in $\oplus_{k=1}^{n} \mathscr{H}$. Then

$$
|\langle\tilde{X} y, y\rangle|=\left|\sum_{k=1}^{n-1}\left\langle X y_{k}, y_{k+1}\right\rangle+\left\langle X y_{n}, y_{1}\right\rangle\right|
$$




$$
\begin{aligned}
& \leq\left(\sum_{k=1}^{n}\left\|y_{k}\right\|^{2}\right) w(X) \quad \text { (by Lemma 2.2) } \\
& =\|y\|^{2} w(X) \\
& =w(X) .
\end{aligned}
$$

It follows, by taking the supremum of the left-hand side of the inequality (2.7) over all unit vectors $y$ in $\oplus_{k=1}^{n} \mathscr{H}$, that

$$
w(\tilde{X}) \leq w(X) .
$$

On the other hand, let $x$ be a unit vector in $\mathscr{H}$, and let $y_{0}=\left[y_{1}, \ldots, y_{n}\right]^{T}$ with $y_{k}=\frac{x}{\sqrt{n}}, k=1, \ldots, n$. Then $y_{0}$ is a unit vector in $\oplus_{k=1}^{n} \mathscr{H}$, and so

$$
\begin{aligned}
w(\tilde{X}) & \geq\left|\left\langle\tilde{X} y_{0}, y_{0}\right\rangle\right| \\
& =\left|\sum_{k=1}^{n-1}\left\langle X y_{k}, y_{k+1}\right\rangle+\left\langle X y_{n}, y_{1}\right\rangle\right| \\
& =\left|\frac{1}{n} \sum_{k=1}^{n-1}\langle X x, x\rangle+\frac{1}{n}\langle X x, x\rangle\right| \\
& =|\langle X x, x\rangle| .
\end{aligned}
$$

It follows, by taking the supremum of the right-hand side of the inequality (2.9) over all unit vectors $x$ in $\mathscr{H}$, that

$$
w(\tilde{X}) \geq w(X) .
$$

Now, the result follows from the inequalities (2.8) and (2.10).

Based on Lemma 2.2, we have the following numerical radius inequalities for several operators.

Theorem 2.4. Let $A_{1}, \ldots, A_{n}, X \in \mathfrak{B}(\mathscr{H})$. Then

$$
w\left(\sum_{k=1}^{n-1} A_{k+1}^{*} X A_{k}+A_{1}^{*} X A_{n}\right) \leq\left(\sum_{k=1}^{n}\left\|A_{k}\right\|^{2}\right) w(X) .
$$

In particular, if $A_{1}, \ldots, A_{n}$ are contractions, then

$$
w\left(\sum_{k=1}^{n-1} A_{k+1}^{*} X A_{k}+A_{1}^{*} X A_{n}\right) \leq n w(X) .
$$


Proof. Let $x \in \mathscr{H}$ be a unit vector, and let $x_{k}=A_{k} x, k=1, \ldots, n$. Then

$$
\begin{aligned}
& \left|\left\langle\left(\sum_{k=1}^{n-1} A_{k+1}^{*} X A_{k}+A_{1}^{*} X A_{n}\right) x, x\right\rangle\right| \\
& \quad=\left|\sum_{k=1}^{n-1}\left\langle X A_{k} x, A_{k+1} x\right\rangle+\left\langle X A_{n} x, A_{1} x\right\rangle\right| \\
& \quad=\left|\sum_{k=1}^{n-1}\left\langle X x_{k}, x_{k+1}\right\rangle+\left\langle X x_{n}, x_{1}\right\rangle\right| \\
& \quad \leq\left(\sum_{k=1}^{n}\left\|x_{k}\right\|^{2}\right) w(X) \quad \text { (by Lemma 2.2) } \\
& \quad=\left(\sum_{k=1}^{n}\left\|A_{k} x\right\|^{2}\right) w(X) \\
& \quad \leq\left(\sum_{k=1}^{n}\left\|A_{k}\right\|^{2}\right) w(X)
\end{aligned}
$$

Now, the result follows by taking supremum of the left-hand side of the inequality (2.11) over all unit vectors $x$ in $\mathscr{H}$.

REMARK 2.5. Another proof of Theorem 2.4 can be seen as follows. Let

$$
\tilde{A}=\left[\begin{array}{cccc}
A_{2} & 0 & \cdots & 0 \\
A_{3} & 0 & \cdots & 0 \\
\vdots & \vdots & & \vdots \\
A_{n} & 0 & \cdots & 0 \\
A_{1} & 0 & \cdots & 0
\end{array}\right]
$$

be $n \times n$ operator matrix in $\mathfrak{B}\left(\oplus_{k=1}^{n} \mathscr{H}\right)$, and let $\tilde{X}$ be as in Proposition 2.3. Then

$$
\begin{aligned}
w\left(\sum_{k=1}^{n-1} A_{k+1}^{*} X A_{k}+A_{1}^{*} X A_{n}\right) & =w\left(\tilde{A}^{*} \tilde{X} \tilde{A}\right) \\
& \leq\|\tilde{A}\|^{2} w(\tilde{X}) \\
& =\left(\sum_{k=1}^{n}\left\|A_{k}\right\|^{2}\right) w(X) \quad \text { (by Proposition 2.3). }
\end{aligned}
$$

In the following result, we present our generalization of the inequality (1.1) to several operators. 
Theorem 2.6. Let $A_{1}, \ldots, A_{2 n}, X \in \mathfrak{B}(\mathscr{H})$. Then

$$
\begin{aligned}
& w\left(\sum_{k=1}^{2 n-1} A_{k+1}^{*} X A_{k}+A_{1}^{*} X A_{2 n}\right) \\
& \leq 2\left(\sum_{k=1}^{n}\left\|A_{2 k-1}\right\|^{2}\right)^{1 / 2}\left(\sum_{k=1}^{n}\left\|A_{2 k}\right\|^{2}\right)^{1 / 2} w(X) .
\end{aligned}
$$

Proof. It follows from Theorem 2.4 that

$$
w\left(\sum_{k=1}^{2 n-1} A_{k+1}^{*} X A_{k}+A_{1}^{*} X A_{2 n}\right) \leq\left(\sum_{k=1}^{2 n}\left\|A_{k}\right\|^{2}\right) w(X) .
$$

In the inequality (2.12), replacing $A_{2 k-1}$ by $t A_{2 k-1}$ and $A_{2 k}$ by $\frac{1}{t} A_{2 k}, t>0$, $k=1, \ldots, n$, we have

$$
w\left(\sum_{k=1}^{2 n-1} A_{k+1}^{*} X A_{k}+A_{1}^{*} X A_{2 n}\right) \leq \frac{t^{4} \alpha+\beta}{t^{2}} w(X),
$$

where $\alpha=\sum_{k=1}^{n}\left\|A_{k}\right\|^{2}$ and $\beta=\sum_{k=1}^{n}\left\|A_{2 k}\right\|^{2}$. Since $\inf _{t>0} \frac{t^{4} \alpha+\beta}{t^{2}}=2 \sqrt{\alpha \beta}$, then the result follows by taking infimum of the right hand side of the inequality (2.13) over all positive real numbers $t$.

A particular case of Theorem 2.6 can be presented as follows. This result shows that Theorem 2.6 is a generalization of the inequality (1.1) to several operators.

Corollary 2.7. Let $A, B, X \in \mathfrak{B}(\mathscr{H})$. Then

$$
w\left(A^{*} X B+B^{*} X A\right) \leq 2\|A\|\|B\| w(X) .
$$

In particular, letting $B=I$, we have

$$
w\left(A^{*} X+X A\right) \leq 2\|A\| w(X) .
$$

Proof. The result follows by applying Theorem 2.6, for $n=1$, to the operators $A_{1}=A$ and $A_{2}=B$.

\section{A refinement of the inequality (1.2)}

The aim of this section is to give a refinement of the Fong-Holbrook inequality (1.2). In their proof of the inequality (1.2), Fong and Holbrook used a result of 
M. J. Crabb (see, e.g., [1, Theorem 3] and [2, Theorem 3.7]). This result says that if $A \in \mathfrak{B}(\mathscr{H})$ such that $w(A) \leq 1$, then

$$
\|A x\|^{2}+\left\|A^{*} x\right\|^{2} \leq 4
$$

for all unit vectors $x \in \mathscr{H}$. In order to give a refinement of the inequality (1.2), we have to refine the inequality (3.1). To do this, we start with the following result.

Lemma 3.1. Let $A, B \in \mathfrak{B}(\mathscr{H})$. Then

(3.2) $\left\|A A^{*}+B B^{*}\right\|$

$$
\leq \max \left(\|A+B\|^{2},\|A-B\|^{2}\right)-\frac{\left|\|A+B\|^{2}-\|A-B\|^{2}\right|}{2} .
$$

In particular, letting $B=A^{*}$, we have

(3.3) $\left\|A A^{*}+A^{*} A\right\|$

$$
\leq 4 \max \left(\|\operatorname{Re} A\|^{2},\|\operatorname{Im} A\|^{2}\right)-2\left|\|\operatorname{Re} A\|^{2}-\|\operatorname{Im} A\|^{2}\right| .
$$

Proof. We have

$$
\begin{aligned}
\max & \left(\|A+B\|^{2},\|A-B\|^{2}\right) \\
& =\max \left(\left\|A^{*}+B^{*}\right\|^{2},\left\|A^{*}-B^{*}\right\|^{2}\right) \\
& =\frac{\left\|A^{*}+B^{*}\right\|^{2}+\left\|A^{*}-B^{*}\right\|^{2}}{2}+\frac{\left|\left\|A^{*}+B^{*}\right\|^{2}-\left\|A^{*}-B^{*}\right\|^{2}\right|}{2} \\
& =\frac{\left\|\left|A^{*}+B^{*}\right|^{2}\right\|+\left\|\left|A^{*}-B^{*}\right|^{2}\right\|}{2}+\frac{\left|\|A+B\|^{2}-\|A-B\|^{2}\right|}{2} \\
& \geq \frac{\left\|\left|A^{*}+B^{*}\right|^{2}+\left|A^{*}-B^{*}\right|^{2}\right\|}{2}+\frac{\left|\|A+B\|^{2}-\|A-B\|^{2}\right|}{2} \\
& =\left\|A A^{*}+B B^{*}\right\|+\frac{\left|\|A+B\|^{2}-\|A-B\|^{2}\right|}{2} .
\end{aligned}
$$

Our refinement of the inequality (3.1) can be stated as follows.

Lemma 3.2. Let $A \in \mathfrak{B}(\mathscr{H})$ such that $w(A) \leq 1$, and let $x$ be a unit vector in $\mathscr{H}$. Then

$$
\|A x\|^{2}+\left\|A^{*} x\right\|^{2} \leq 4\left(1-\frac{\left|\|\operatorname{Re} A\|^{2}-\|\operatorname{Im} A\|^{2}\right|}{2}\right) .
$$


Proof. It follows from the inequality (3.3) that

$$
\begin{aligned}
\| A x & \left\|^{2}+\right\| A^{*} x \|^{2} \\
& =\left|\left\langle\left(A A^{*}+A^{*} A\right) x, x\right\rangle\right| \\
& \leq\left\|A A^{*}+A^{*} A\right\| \\
& \leq 4 \max \left(\|\operatorname{Re} A\|^{2},\|\operatorname{Im} A\|^{2}\right)-2\left|\|\operatorname{Re} A\|^{2}-\|\operatorname{Im} A\|^{2}\right| \quad(\text { by Lemma 3.1) } \\
& =4 \max \left(w^{2}(\operatorname{Re} A), w^{2}(\operatorname{Im} A)\right)-2\left|\|\operatorname{Re} A\|^{2}-\|\operatorname{Im} A\|^{2}\right| \\
& \leq 4 w^{2}(A)-2\left|\|\operatorname{Re} A\|^{2}-\|\operatorname{Im} A\|^{2}\right| \\
& \leq 4\left(1-\frac{\left|\|\operatorname{Re} A\|^{2}-\|\operatorname{Im} A\|^{2}\right|}{2}\right) .
\end{aligned}
$$

Based on Lemma 3.2, we have the following general numerical radius inequality.

Theorem 3.3. Let $A, B, X, Y \in \mathfrak{B}(\mathscr{H})$. Then

(3.5) $w(A X B \pm B Y A)$

$$
\leq 2 \sqrt{2}\|B\| \max (\|X\|,\|Y\|) \sqrt{w^{2}(A)-\frac{\left|\|\operatorname{Re} A\|^{2}-\|\operatorname{Im} A\|^{2}\right|}{2}} .
$$

Proof. First, suppose that $w(A) \leq 1,\|X\| \leq 1,\|Y\| \leq 1$, and let $x$ be a unit vector in $\mathscr{H}$. Then

$$
\begin{aligned}
|\langle(A X \pm Y A) x, x\rangle| & =\left|\left\langle X x, A^{*} x\right\rangle \pm\left\langle A x, Y^{*} x\right\rangle\right| \\
& \leq\|X x\|\left\|A^{*} x\right\|+\|A x\|\left\|Y^{*} x\right\| \\
& \leq\left\|X^{*}\right\|\left\|A^{*} x\right\|+\|A x\|\left\|Y^{*}\right\| \\
& \leq\left\|A^{*} x\right\|+\|A x\| \\
& \leq \sqrt{2}\left(\left\|A^{*} x\right\|^{2}+\|A x\|^{2}\right)^{1 / 2} \\
& \leq 2 \sqrt{2} \sqrt{1-\frac{\left|\|\operatorname{Re} A\|^{2}-\|\operatorname{Im} A\|^{2}\right|}{2}} \quad \text { (by Lemma 3.2), }
\end{aligned}
$$

and so

$$
\begin{aligned}
w((A X \pm Y A)) & =\sup _{\|x\|=1}|\langle(A B \pm B A) x, x\rangle| \\
& \leq 2 \sqrt{2} \sqrt{1-\frac{\left|\|\operatorname{Re} A\|^{2}-\|\operatorname{Im} A\|^{2}\right|}{2}} .
\end{aligned}
$$


For the general case, let $A, X$, and $Y$ be any operators in $\mathfrak{B}(\mathscr{H})$. It is clear that the result is trivial if $w(A)=0$ or $\max (\|X\|,\|Y\|)=0$, so suppose that $w(A) \neq 0$ and $\max (\|X\|,\|Y\|) \neq 0$. In the inequality (3.6), replacing the operators $A, X$, and $Y$ by the operators $\frac{A}{w(A)}, \frac{X}{\max (\|X\|,\|Y\|)}$, and $\frac{Y}{\max (\|X\|,\|Y\|)}$, respectively, we have

$$
\begin{aligned}
w(A X & \pm Y A) \\
& \leq 2 \sqrt{2} \max (\|X\|,\|Y\|) w(A) \sqrt{1-\frac{\left|\left\|\operatorname{Re}\left(\frac{A}{w(A)}\right)\right\|^{2}-\left\|\operatorname{Im}\left(\frac{A}{w(A)}\right)\right\|^{2}\right|}{2}} \\
& =2 \sqrt{2} \max (\|X\|,\|Y\|) \sqrt{w^{2}(A)-\frac{\left|\|\operatorname{Re} A\|^{2}-\|\operatorname{Im} A\|^{2}\right|}{2}} .
\end{aligned}
$$

Now, in the inequality (3.7), replacing the operators $X$ and $Y$ by $X B$ and $B Y$, respectively, we have

$$
\begin{aligned}
& w(A X B \pm B Y A) \\
& \leq 2 \sqrt{2} \max (\|X B\|,\|B Y\|) \sqrt{w^{2}(A)-\frac{\left|\|\operatorname{Re} A\|^{2}-\|\operatorname{Im} A\|^{2}\right|}{2}} \\
& \quad \leq 2 \sqrt{2}\|B\| \max (\|X\|,\|Y\|) \sqrt{w^{2}(A)-\frac{\left|\|\operatorname{Re} A\|^{2}-\|\operatorname{Im} A\|^{2}\right|}{2}},
\end{aligned}
$$

as required.

An application of Theorem 3.3 can be seen as follows. This result contains our promised refinement of the inequality (1.2).

Corollary 3.4. Let $A, B \in \mathfrak{B}(\mathscr{H})$. Then

$$
w(A B \pm B A) \leq 2 \sqrt{2}\|B\| \sqrt{w^{2}(A)-\frac{\left|\|\operatorname{Re} A\|^{2}-\|\operatorname{Im} A\|^{2}\right|}{2}}
$$

and

$$
w\left(A^{2}\right) \leq \sqrt{2}\|A\| \sqrt{w^{2}(A)-\frac{\left|\|\operatorname{Re} A\|^{2}-\|\operatorname{Im} A\|^{2}\right|}{2}} .
$$

Proof. The inequality (3.8) follows from Theorem 3.3 by letting $X=Y=$ $I$, while the inequality (3.9) follows from the inequality (3.8) by letting $B=A$.

An application of Corollary 3.4 can be stated as follows.

Corollary 3.5. Let $A, B \in \mathfrak{B}(\mathscr{H})$ such that $w(A B+B A)=2 \sqrt{2}\|B\|$. $w(A)$ or $w(A B-B A)=2 \sqrt{2}\|B\| w(A)$. Then $\|\operatorname{Re} A\|=\|\operatorname{Im} A\|$. 
Proof. Suppose that $w(A B+B A)=2 \sqrt{2}\|B\| w(A)$ or $w(A B-B A)=$ $2 \sqrt{2}\|B\| w(A)$. Since

$$
\begin{aligned}
w(A B \pm B A) & \leq 2 \sqrt{2}\|B\| \sqrt{w^{2}(A)-\frac{\left|\|\operatorname{Re} A\|^{2}-\|\operatorname{Im} A\|^{2}\right|}{2}} \\
& \leq 2 \sqrt{2}\|B\| w(A),
\end{aligned}
$$

it follows that

$$
\sqrt{w^{2}(A)-\frac{\left|\|\operatorname{Re} A\|^{2}-\|\operatorname{Im} A\|^{2}\right|}{2}}=w(A)
$$

and so $\|\operatorname{Re} A\|=\|\operatorname{Im} A\|$.

\section{REFERENCES}

1. Ando, T., Structure of operators with numerical radius one, Acta Sci. Math. (Szeged) 34 (1973), 1-15.

2. Ando, T., Topics in Dilation Theory, Sapporo, 1973.

3. Halmos, P. R., A Hilbert Space Problem Book, 2ed, Grad. Texts Math. 19, Springer, New York 1982.

4. Hirzallah, O., Kittaneh, F., and Shebrawi, K., Numerical radius inequalities for commutators of Hilbert space operators, Numer. Funct. Anal. Optim. 32 (2011), 739-749.

5. Hirzallah, O., Kittaneh, F., and Shebrawi, K., Numerical radius inequalities for certain $2 \times 2$ operator matrices, Integral Equations Operator Theory 71 (2011), 129-147.

6. Fong C.-K., and Holbrook, J. A. R., Unitarily invariant operator norms, Canad. J. Math. 35 (1983), 274-299.

DEPARTMENT OF MATHEMATICS

HASHEMITE UNIVERSITY

ZARQA

JORDAN

E-mail: o.hirzal@hu.edu.jo
DEPARTMENT OF MATHEMATICS THE UNIVERSITY OF JORDAN

AMMAN

JORDAN

E-mail: fkitt@ju.edu.jo 